\title{
EL DOMINIO RACIONAL DEL SIGLO XIII. CONSIDERACIÓN HISTÓRICO-EPISTEMOLÓGICA
}

\author{
María Angeles Vitoria \\ Licenciada en Biología. Dra. en CC. Educación Castelgandolfo (Roma)
}

A lo largo de la historia, filosofía, ciencia y teología han asumido sucesivas evoluciones, ampliaciones y restricciones. Cada época se ha caracterizado por un peculiar entrelazamiento de estos órdenes en el que primaba uno u otro saber.

En la antigüedad, la distinción entre filosofía y ciencias, apenas esbozada y no tematizada, se mantenía fundamentalmente como una distinción material en el sentido de que la filosofía estudiaba las cuestiones más importantes y primarias, y las ciencias las más particulares.

El periodo medieval es, desde esta perspectiva epistemológica, heterogéneo pero, en líneas generales, puede decirse también que la ciencia vivía inmersa en una atmósfera filosófico-teológica. ${ }^{1}$ Dentro de este vasto periodo dirigimos la atención al siglo XIII, época en la que -como justificaremos seguidamente- se da una situación de privilegio para una reflexión fecunda acerca de las consecuencias de una unidad del saber garante de su diversidad. Este es el hilo conductor de cuanto exponemos.

\section{I. ÁMBITOS DE LA RACIONALIDAD}

Comenzamos con unas breves consideraciones introductorias.

En el ámbito del saber humano el funcionamiento riguroso de la inteligencia -su proceder hacia el logro de un saber demostrativo- se realiza fundamentalmente a través de dos perspectivas diversas: filosofía y ciencias particulares. Mientras las ciencias -naturales 0 humanas- operan sobre sectores específicos de la realidad física y espiritual, la filosofía

1 Esta afirmación, con los debidos matices, seguirá teniendo validez hasta el siglo XVII, momento en el que configura de modo definitivo el método experimental-matemático. 
mantiene la mirada dirigida a la totalidad de aquello con lo que se encuentra, tratando de entender la realidad en cuanto tal, su significado último. ${ }^{2}$

Esta distinción de órdenes en el saber no es circunstancial ni fortuita, fruto de una particular coyuntura cultural. Se trata de una diferenciación intrínseca, «consecuencia necesaria del modo de conocer del hombre, que debe siempre asumir el punto de vista de los principios generales y, como desde abajo, el análisis de lo particular». ${ }^{3}$ Filosofía y ciencias se sitúan, pues, en planos abjetuales diversos, singularizándose tanto por el ámbito de la realidad al que cada una se dirige-principios y objeto formal-como por el método o modo de establecer contacto con él.

Aunque la pluriforme variedad del saber científico incluye las ciencias humans y las llemadas positivas, en adelante, al referimos a las ciencia, entendermos las de método experimental-matemático; y bajo el concepto de filosofía connotaremos principalmente la metafísicas que constituye su núcleo.

La distinción de la que venimos hablando -ahora ya la distinción entre filosofía y ciencias de método experimental matemático- ha sido muy discutida y envuelve en sí buena parte de la problemática de la filosofía de las ciencias. ${ }^{4}$

Dejando de lado la interesante cuestión de la demarcación de sus respectivas autonomías, nos fijaremos en otro aspecto reladionado con éste y no de menor interés: el de la integración de ambos saberes. En efecto, el proceder científico y filosófico nos van progresivamente desvelando los estractos fenoménicos y las intimidades del ser, ${ }^{5}$ aspectos que, dándose unidos en la realidad, nos llegan de modo fragmentario, aislado, atomizado. Atribuir a cada cosa su realidad es exigencia ineludible del conocimiento verdadero: veritas est adaequatio rei et intellectus. ${ }^{6} \mathrm{Y}$ puesto que pertenece a la naturaleza del entendimiento conformarse con las cosas, el acto de unificación y síntesis de los datos recibidos se presenta en cada orden del conocer y en el conocer en su conjunto. Observamos así en las fases de construcción de cada sector científico un movimiento circular en el que las observaciones experimentales desembocan en leyes y éstas, a su vez, en una sucesión de teorías que abarcan y explican un número cada vez mayor de fenómenos. ${ }^{7}$

También ciencias y filosofía tienden a converger en el conocimiento analógico de la misma realidad, aunque, en este caso, la articulación de saberes es más delicada y difícil: la cosmovisión científico-filosófica ha de respetar rigurosamente los dos ámbitos epistemológicos,

2 Piepper, J., Filosofía medival y mundo moderno, Rialp, Madrid, 1979, p. 375. Selvaggi, F., Filosofia delle scienze, Edic. Civiltà Cattolica, Roma, 1953, pp. 311-331.

3 Sanguineti, J.J., Ciencia y Modernidad, Lohlé, Buenos Aires, 1988, pp. 41-41.

4 Los términos en los que E. Agazzi expone la cuestión; nos parecen particularmente lúcidos. Véanse, entre otras obras, Agazzi, E., Temi e problemi di filosofia della fisica, Ed. Abate, Roma, 1974; L'epistemologia contemporanea: il concetto attuale di scienza en AA.VV., Scienza e Filosofia oggi, Ed. Massimo, Milano, 1980, pp. 7-20; Scienza e Metafisica, «Per la Filosofia», I/2 (1984) 1-13; Philosophie, Science, Metaphysique, Ed. Univ., Fribourg, Suisse, 1987.

5 Fabro, C., Percezione e Pensiero, Ed. Morcelliana, Brescia, 1962, pp. 614-615.

6 Santo Tomás, De Verit., q. 1, a. 1. Si no se indica lo contrario, se ha utilizado la edición Marietti.

7 Artigas, M., Sanguineti, J.J., Filosofía de la naturaleza, Eunsa, Pamplona, 1989, pp. 236-243. 
de modo que el poder unificante de la filosofía no desemboque en una unidad monista, sino que, al mostrar el anclaje de los saberes científicos en el ser, ponga de relieve la verdad propia de cada sector o faceta considerada, determinando el lugar que debe ocupar en el conjunto del conocer.

Hasta el momento nos hemos mantenido en el dominio del saber humano-natural. Sin embargo, la consideración completa del horizonte cognoscitivo incluye otro orden de conocimiento, que presupone el natural. ${ }^{8}$ Sabemos por la fe que existe, además del orden natural de verdades, otro orden -sobrenatural- que excediendo la razón humana, no está en contradicción con ella. ${ }^{9} \mathrm{La}$ fe cristiana tiene su origen en la Revelación con la que Dios manifestó, junto con misterios sobrenaturales, las verdades naturales más altas que se refieren a Dios y a las relaciones del hombre con Dios.

Con la dimensión de la fe se ha abierto un nuevo marco a la racionalidad humana, también en su vertiente científica, es decir, teológica: el creyente puede progresar en el entendimiento de las verdades sobrenaturales descubriendo sus relaciones por medio de razonamientos, y esta exposición de lo contenido virtualmente en la fe es precisamente la ciencia teológica, que requiere el uso filosófico de la razón: ${ }^{10}$ «la teología se hace por y desde la fe con la filosofía». ${ }^{11}$

El dominio de la racionalidad teológica está llamado también a integrarse con los otros conocimientos naturalmente alcanzados pero sin subsumir la totalidad del saber. ${ }^{12}$ Una realidad sin compartimentos estancos tiene como correlato cognoscitico la integración de verdades: cada verdad debe difundir su luz sobre todas las demás; cada verdad ha de contribuir, desde su lugar, a hacer inteligible el universo.

\section{ELEMENTOS DE LA INTERDISCIPLINARIEDAD TOMISTA}

Expuestas esquemáticamente lo que consideramos las coordenadas básicas de la racionalidad, retrocedemos siete siglos para extraer de la producción científico-filosófica y teológica de Santo Tomás enseñanzas de fecundidad interdisciplinar. Al hacerlo, no vamos en pos de una síntesis definitiva de saberes, sino en búsqueda del espíritu y de los fundamentos sólidos para -generación tras generación- continuar edificándola con éxito.

Tratamos de mostrar, en primer ligar, que el planteamiento tomista del saber es lo suficientemente amplio, profundo y articulado como para incluir en su seno una modalidad de conocimiento que en su tiempo todavía no se había formalmente producido: la ciencia

8 Santo Tomás, De Verit., q. 14, a. 9, ad 8.

9 Idem, C.G. I, c. 3, y Commentum in Libros IV Sententiarum, Ludovicum Vives, Parissiis, 1878. In III Sent., d. 24, q. 1, a. 3, sol. 2, ad 2.

10 Idem, In I Sent. Prol. q. 1, a. 3.

11 Cardona, C., Metafísica de la opción intelectual, Ed. Rialp, Madrid, 1973, p. 261.

12 Illanes, J.L., Teología y ciencias en una visión cristiana de la Universidad, en Scripta Theologica 14 (1982/3) 873-888. 
experimental. Y concluímos la exposición poniendo de manifiesto el fundamento ontológico y epistemológico que sustenta esa total apertura a todo lo verdadero, y sus consecuencias.

El siglo XIII, más conocido por sus felices síntesis filosófico-teológicas, es también escenario de auténtica producción científica, sobre todo, de fecundas directrices metodológicas para su desarrollo. ${ }^{13}$

La recepción de las obras de Aristóteles, de Euclides, de la ciencia griega y árabe, robusteció la dimensión racional de la filosofía y de la matemática que, unida al empirismo de la técnica, engendró una nueva ciencia empírica que trataba de descubrir la estructura racional de la naturaleza. A partir de entonces, la historia de la ciencia medieval no es sino elaboración de este nuevo modo de abordar la naturaleza; cuyo método será empleado, por primera vez, y con plena madurez y eficiacia por Galileo. ${ }^{14}$

Desde una perspectiva epistemológica, puede decirse que destacan dos contribuciones. La recuperación de la idea de ciencia como saber demostrativo, como explicación racional (un hecho concreto es explicado cuando puede ser deducido de un principio más general), que se produjo gracias a la recuperación gradual de abordar la ciencia característico de los árabes: $n o$ para ver qué aspectos de la naturaleza ilustraban más vivamente las intenciones morales, $n i$ cuáles eran las causas naturales que podían proporcionar una explicación racional de los hechos descritos en la Biblia y observados en el mundo de la experiencia cotidiana, sino qué tipo de conocimiento podía dar el dominio de la naturaleza. ${ }^{15}$

Santo Tomás recibió este patrimonio y, amante de la verdad como pocos, no se mantuvo al margen de tales acontecimientos. Conocedor profundo de la ciencia de su época, aunque no dedicó sus esfuerzos al desarrollo de este sector del saber, destacan sus contribuciones epistemológicas sobre la naturaleza y el método de las ciencias.

En la concepción aristotélico-tomista, la ciencia, en sentido amplio, es cognitio certa per causas. Se entiende la ciencia como saber que, partiendo de principios evidentes, universales o particulares, obtiene por vía lógico-demostrativa conclusiones ciertas. La certeza y el carácter definitivo de las conclusiones viene dado por el punto de partida -principios evidentes-y por el rigor de la lógica deductiva.

A primera vista, este ideal científico, aplicado a las ciencias de método experimentalmatemático, parece no tener nada en común con lo que sucede realmente en la ciencia moderna, cuya característica fundamental es que la justificación de las premisas no se hace sobre la base

13 Duhen ha dedicado muchas páginas a la descripción de la producción científica del medioevo y especialmente a manifestar la continuidad del progreso científico en la época medieval y la continuidad entre la ciencia medieval y moderna. Duhem, P., Le système du monde, 10 vol. Ed. Hermann, Paris, 1913-14. Jaki, S.L., The Road of Science and the Ways to God, Scottish Academic Press, Edimburgh, 1978; y los conocidos estudios de Maier, A., Scienza e filosofia nel medioevo (saggi sui secoli XIII e XIV), Ed. Jaca Book, Milano, 1983.

14 Crombie, A.C., Historia de la ciencia. De San Agustín a Galileo, Vol. I, Ed. Alianza, Madrid, 1987, pp. 21-22 y $47-54$.

15 Crombie, A.C., op. cit., vol, I, P.57; Ibidem, Vol. II, P. 13. 
de una evidencia a priori, sino sobre la base de la conformidad de sus conclusiones con la experiencia. $^{16}$

Esta y otras constataciones han llevado a contraponer la concepción aristotélico-tomista de la ciencia con la moderna, declarando un vaniloquio todo intento de buscar en aquella otientaciones útiles para la articulación de los saberes filosófico y teológico. La cuestión adquiere, sin embargo, matices y aclaraciones.

Ciertamente no encontramos en Santo Tomás una ciencia positiva elaborada, ni en cuanto a sus contenidos ni en lo que refiere a determiaciones metódicas particulares. El ideal de la ciencia como conocimiento demostratrivo por medio de causas verdaderas no basta para dar cuenta del alcance de las demostraciones de la ciencia actual. ${ }^{17}$ Pero queda como perennemente valioso su noción de ciencia como conocimiento mediato argumentado, con bases en postulados y basado en la experiencia. Además, su distinción entre metafísica y ciencias particulares posee también un significado orientativo para la distinción e integración moderna entre filosofía y las ciencias positivas. Veamos más detalladamente estos aspectos.

Los escritos de Santo Tomás muestran apertura hacia una racionalidad plural. Reconoce la existencia de un saber hipotético deductivo que no produce la certeza del fundado en principios evidentes: A veces, empero, la inquisición de la razón no puede conducir hasta el último término (esto es, hasta el intellectus principiorum) sino que permanece en la inquisición misma, y entonces al investigador le quedan vías abiertas hacia distintas soluciones; y esto ocurre cuando se procede por razones probables. ${ }^{18}$

En la Summa Theologica se contiene otro texto significativo: para explicar algo se pueden aducir dos tipos de razones; unas prueban una tesis suficientemente (...). Otras no lo hacen, sino que se limitan a mostrar la congruencia con una serie de efectos; así, en la astronomía, se acude a los excéntricos y epiciclos, de modo que a patir de esta hipótesis se salvan las apariencias de los movimientos celestes, pero esta tesis no está sufientemente probada, pues esos fenómenos quizá podrían explicarse con otra hipótesis..$^{19}$

Aunque encontramos en las obras de Santo Tomás afirmaciones puntuales en las que parece inclinarse hacia una consideración no científica de lo que hoy entendemos por ciencia

16 Properi, G.M., L'Imagine fisica del mondo. «Per la Filosofia» I (1984/2) 35-44; Sanguineti, J.J., Ciencia y Modernidad, p. 185; Vid. Manara, C.F., Metodi della scienza dal Rinascimento ad oggi, Ed. Vita e Pensiero, Milano, 1975.

17 No es objeto del presente estudio reflexionar sobre las pautas de validez de los enunciados y demostraciones de la ciencia moderna, sino determinar hasta qué punto en la visión de la ciencia de Santo Tomás podemos encrontar indicaciones útiles para la actual integración de los saberes. Remitimos al lector interesado en el tema a la reciente publicación de M. Artigas, Filosofía de la ciencia experimental, Eunsa, Pamplona, 1989.

18 Santo Tomás, in librum Boethii de Trinitate expositio, Opuscula Varia (Vol. XXVIII), Ludovicum Vives, Parisiis, 1875. In Boeth. de Trin., q. 6, a. 1. Santo Tomás concluye el texto afirmando que esta vía produce opinión o fe (humana), no ciencia, pero al oponer este modo de proceder al proceso demostrativo, entendemos que piensa en la demostración perfecta o propter quid. En otro lugar (In II Coelo et Mundo, lec. 7), refiriéndose a cuestiones astronómicas afirma que sobre estos temas de los que se tiene escasa experiencia, es suficiente decir lo que nos parece, aunque no se tenga completa certeza.

19 Santo Tomás, S.Th. I, q. 32, a. 1, ad 2. Pueden consultarse ejemplos concretos In II De Coelo et Mundo, lect. 4; In II De Coelo et Mundo, lect. 11. 
física, la doctrina recogida en las cuestiones 5 y 6 del Comentario al De Trinitate de Boecio en la que propone tres niveles fundamentales de inteligibilidad de lo real llevan a presumir lo contrario. $^{20}$

En la perspectiva tomista, la realidad puede estudiarse según tres enfoques básicos: la intelección física, propia de las ciencias de la naturaleza; la intelección matemática peculiar de la ciencia del mismo nombre; y la intelección metafísica característica de la filosofía. ${ }^{21}$

La doctrina de los tres niveles de abstracción -el metafísico más propiamente separationo es interpretado por Santo Tomás de un modo cerrado: caben movimientos de uno a otro nivel y situaciones intermedias. Concretamente, el nivel de intelección físico-matemático, característico de la física moderna, aunque con una formalización diversa, era ya conocido en la época de Aristóteles y mereció una consideración atenta por parte de Santo Tomás, si bien personalmente no dedicó sus energías a desarrollar una ciencia siguiendo este ideal metódico. ${ }^{22}$

La mayor flexibilidad de la noción tomista de ciencia en relación con la aristotélica, consecuencia de la concepción analógica del ser y de la verdad, lleva a Santo Tomás a advertir el defecto aristotélico de una consideración excesivamente unívoca de la ciencia, admitiendo la posibilidad de saberes con distinto alcance demostrativo: quizá la apariencia de las estrellas pueda explicarse por algún otro modo, aún no comprendido por los hombres. Sin embargo, Aristóteles utiliza estas suposiciones como verdaderas, al tratar de las cualidades de los movimientos. ${ }^{23}$

Como señalamos anteriormente, no se encuentra en la doctrina tomista una exposición acabada de la naturaleza y alcance del método experimental-matemático que, en sus tiempos, no era todavía algo sistemáticamente constituído, pero sí se descubren indicaciones valiosas para la síntesis filosófico-científica en la que puede entrar con pleno derecho la ciencia actual.

La distinción de los enfoques básicos de estudio de la realidad lleva a caracterizar las ciencias por el grado de separación de la materia que, siendo principio de coartación de la actividad del ente, es también causa de su menor inteligibilidad. De ahí que se puedan distinguir ordenadamente los saberes científicos, según el modo en que dejan de lado la materia en su consideración, sin que pierdan unidad. ${ }^{24}$

20 El opúsculo Expositio super librum Boethii De Trinitate, ha sido definido por Grabmann como «el tratado más detallado y profundo de la doctrina tomista de la ciencia» (M.Grabmann, Il concetto di scienza secondo $S$. Tommaso d'Aquino e le relazioni della fede e della teologia con la filosofia e le scienze profane, Revista di Filosofia Neoescolastica 26 (1934). Para una traducción y comentarios de esta obra del Aquinate véase A. García Marqués y J.A. Fernández, Santo Tomás de Aquino. Exposición del De Trinitate de Boecio, Eunsa, Pamplona, 1986. Véase también L. Elders, Faith and Science, Ed. Herder, Roma, 1974.

21 Santo Tomás expone esta doctrina en In Boeth. De Trin. qq. 5 y 6 ; también In VI Metaph., lect. 1, nn 11561165. Para una excelente exposición comentada, consúltese J.J. Sanguineti, La Filosofía de la ciencia según Santo Tomás, Eunsa, Pamplona, 1977, cap. III. Véase también R. Masi, Física, matemática, metafísica, Rivista di Filosofia neoscolastica 44 (1952) 109-126.

22 Santo Tomás, In II Phys., lect. 3; In I Anal. Post., lect. 25; In Boeth. de Trin., q. 5, a. 3, ad 6.

23 Santo Tomás, In II De Coelo et Mundo, lect. 17.

24 Selvaggi, F., San Tommaso e la mentalità scientifica moderna, Seminarium 29 (1977) 855-877. Véase también del mismo autor, Evoluzione del cocetto di scienza e dell' epistemologia, Seminarium 26(1974) 491-517. 
La doctrina de los niveles de abstracción cobra vigor -se sustenta- dentro del marco de una concepción metafísica que tiene como clave la noción de acto de ser, entendido como acto fundante del ente y de todo lo que en el ente tiene realidad. ${ }^{25}$ La noción intensiva de acto de ser, clave de la merfísica tomista, es el fundamento último de la unidad y diversidad del saber: siendo los aspectos estudiados por las ciencias particulares aspectos de un ente que tiene una unidad conferida por su acto de ser, es esta misma unidad del ente la que, en último término, exige la unificación y sustentación de las ciencias en la metafísica. ${ }^{26}$ Una síntesis en la que la metafísica abarca todas y cada una de las ciencias sin absorberlas ni sustituirlas, y sin constituirlas en una de sus partes.

El ser como acto fundante de toda la realidad es también el fundamento de la esencial apertura del tomismno a toda la verdad: en el ser cabe todo conocimiento válido, precisamente porque es la perfección de toda perfección y la fuente radical de toda inteligibilidad. ${ }^{27}$

La amplitud de la síntesis cognoscitiva de Santo Tomás, incluye el orden de lo sobrenatural que, distinguiéndose realmente del natural, se integra con él. ${ }^{28}$ Puesto que fuera del ser no hay nada, y lo sobrenatural también es, el ser se constituye en el punto de contacto o de comunicación entre lo natural y lo sobrenatural. Esta noción omnicomprensiva del ser ofrece el fundamento para integrar el conocimiento de realidades obtenido por la fe con otros conocimientos naturalmente alcanzados, a través de la noción misma de realidad, es decir, mediante el acto de ser. ${ }^{29}$

\section{CONSECUENCIAS DE LA AUSENCIA Y PRESENCIA DEL EQUILIBRIO INTERDISCIPLINAR}

El desarrollo completo de la racionalidad, aunque nunca acabado y susceptible siempre de progreso, comporta el cultivo de sus tres direcciones fundamentales: filosofía, ciencias y teología. Y no como ámbitos heterogéneos, sino como saberes relacionados entre sí: con unidad de jerarquía y orden.

A la racionalidad medieval faltó, en cuanto a contenidos, el núcleo bien desarrollado de las ciencias de método experimental-matemático. Podemos decir que quedó truncada en un aspecto que, poseyendo gran valor y utilidad, no es absolutamente necesario para el fin esencial de la vida humana.

La racionalidad moderna -nos referimos a las líneas dominantes de lo que se ha denominado modernidad-disponiendo ya del saber científico-experimental bien constituído,

25 Fabro, C., La nozione metafisica di partecipazione, S.E.I., Torino, 1960; A.L. González, Ser y participación, Eunsa, Pamplona, 1979; E. Gilson, L'essere e l'essenza, Ed. Massimo, Milano, 1988.

26 Santo Tomás, In IV Metaph., lect. 5. Vid. J.J. Sanguineti, La filosofia de la ciencia, según Santo Tomás, pp. 319-153.

27 Cardona, C., Metafisica de la opción intelectual, p. 181.

28 Santo Tomás, In Boeth. De Trin., 1. 1; S.Th. I, q. 36, a. 2.

29 Cardona, C., op. cit., p. 14. 
poseía los elementos convenientes para una síntesis cabal. Sin embargo, el preyecto de la modernidad elegió otros rumbos: proclamó la total autonomía de las ciencias, expulsando la metafísica junto con la fe y la teología del dominio de lo reacional. Pero mientras la ausencia medival fue carencia de algo todavía no logrado, la privación moderna hả sido senda elegida.

Cabe preguntarse por las razones no puramente teoréticas de esta elección acudiendo a las motivaciones presentes en el hombre, artífice y creador de la ciencia. Se ha llamado la atención del método físico-matemático, cuando el mundo de los fenómenos parecía rendirse al empuje de la ciencia. Hasta bien entrado el siglo XIX, los grandes éxitos logrados por la ciencia, llevaron a considerarla, en el plano cognoscitivo, como la forma de saber dotada de plena verdad y absoluta certeza. La dedicación masiva al estudio de los aspectos cuantitativos (los únicos cognoscibles mediante el método experimental-matemático), omitiendo consideraciones filosófico-teológicas, dejaba más fácilmente abierta la posibilidad de pensar que aquellos aspectos eran lo único real: es fácil pasar de la omisión de los otros aspectos-omisión que condiciona el carácter científico de la investigación- a su negación: pasar de su no significación científica a su no significación ontológica. ${ }^{30}$ Ya Santo Tomás, a propósito del reduccionismo matemático de los pitagóricos, advertía que es frecuente entre los hombres la pretensión de juzgar de las cosas a partir de sus descubrimientos. ${ }^{31}$

Los hallazgos científicos parecían confirmar que se había dado con la clave de lectura de la naturaleza, con el método que daría al hombre, en un futuro, el pleno dominio sobre las cosas. La ciencia bastaba, y esta fe sustancialmente dogmática en la omnipotencia de la ciencia se extendió a todos los ámbitos de la vida humana: la ciencia se presentaba como ideal de unión para la humanidad contemporánea, como camino que conduciría a las más altas cotas de paz y felicidad.

El monopolio del dominio racional por parte de la ciencia no tarđó en manifestar sus consecuencias. Los descubrimientos físicos y los estudios matemáticos de finales del XIX y comienzos del XX, provocaron la crisis de la concepción de la ciencia que se había difundido, y el estallido de la Primera Guerra Mundial derribó tạmbién las esperanzas que se habían puesto en ella.

Los hechos mencionados no suponen dejar de reconocer las mejoras enormes de las condiciones de la vida humana que la ciencia y la técnica han procurado, pero sí la constatación de los potenciales efectos destructores que encierran: sin el conocer sapiencial-filosófico y teológico- al que corresponde establecer el lugar que cada ciencia debe ocupar en el conjunto del conocer y del existir humanos, detallando los criterios ético-sapienciales que deben regular su uso, ${ }^{32}$ la ciencia no encamina al hombre a su verdadero bien. Las consecuencias negativas de un dominio poco sabio de la naturaleza son ahora más evidentes, a la par que constituyen una invitación para reflexionar sobre el alcance de la ciencia y sus significado humano, abriendo el camino a la guía de un saber superior.

30 Fabro, C., Historia de la Filosofía, Vol. II, Ed. Rialp, Madrid, 1965, p. 30.

31 Santo Tomás, In I Metaph. lect. 7, n. 120; In De Coelo et Mundo lect. 20, n. 482.

32 Illanes, J.L., Sobre el saber teológico, Ed. Rialp, Madrid, 1978, p. 118. 
Mientras la ciencia vivió inmersa en una atmósfera teológico-filosófica, fue consciente de sus límites, dejándose encuadrar en un campo de especulación más amplio, asumiendo así la función que le correspondía. ${ }^{33}$ Pero, perdido este marco, aparece enseguida, de modo espontáneo, una filosofía que trata de justificar que ese conocimiento científico non era parcial, que no está llamado a completarse con otros conocimientos. Y esta filosofía no puede ser obviamente la filosofía realista, abierta de suyo a los diversos órdenes del ser y, por tanto, del conocer. Esta filosofía no puede ser más que una filosofía trascendental en el sentido kantiano, porque cuando el objeto abstracto de una ciencia no es visto como parte de una realidad más amplia contempable por la inteligencia, el objeto no remite sino al sujeto como función de síntesis de los objetos.

Con notable agudeza ha indicado Gilson la conveniencia educativa de cultivar saberes que nos superan, mostrando que el cultivo de la metafísica y la consideración de las verdades de la fe tienen, entre otros efectos, preservar el conocimiento científico y su uso técnico de caer en la ideología. ${ }^{34}$

33 Sanguineti, J.J., Il destino storico dei valori scientifici, II Nuovo Areopago 1 (1982/2) 15.

34 Gilson, E., Introduction a la philosophie chrétienne, Ed. Vrin, Paris, 1960, p. 118. Vid F. Botturi, Desiderio e verità. Per una antropologia christiana nell' età secolarizzata, Ed. Massimo, Milano, 1985, Parte I. 